\title{
Interference Cancellation and Joint Decoding for Collision Resolution in Slotted ALOHA
}

\author{
Stephan Pfletschinger*, Monica Navarro*, Giuseppe Cocco $^{\dagger}$ \\ ${ }^{*}$ Centre Tecnològic de Telecomunicacions de Catalunya (CTTC) \\ Av. Carl Friedrich Gauss 7, 08860 Castelldefels, Spain \\ ${ }^{\dagger}$ German Aerospace Center - DLR \\ Oberpfaffenhofen, 82234 Wessling, Germany \\ \{stephan.pfletschinger, monica.navarro\}@cttc.es, giuseppe.cocco@dlr.de
}

\begin{abstract}
We present a novel decoding scheme for slotted ALOHA which is based on concepts from physical-layer network coding (PNC) and multi-user detection (MUD). In addition to recovering individual user packets from a packet collision as it is usually done with MUD, the receiver applies PNC to decode packet combinations that can be used to retrieve the original packets using information available from other slots. We evaluate the novel scheme and compare it with another scheme based on PNC that has been proposed recently and show that both attain important gains compared to basic successive interference cancellation. This suggests that combining PNC and MUD can lead to significant gains with respect to previously proposed methods on either one or the other.
\end{abstract}

\section{INTRODUCTION}

In a random multiple-access scheme, various users access the same channel with no or very limited coordination which may result in packet collisions. In contrast to demand assignment multiple-access (DAMA) schemes, in which collisions are avoided by pre-allocating the radio resources to the users, in random multiple-access protocols, such as e.g. ALOHA, packet collisions cannot be avoided due to the lack of coordination. Such uncoordinated multiple-access protocols are preferable over scheduled access in various scenarios including

- systems with long inherent feedback delay, e.g. satellite networks

- networks with a large user population, e.g. wireless sensor networks or RF-IDs tags

- systems with sporadic user activity patterns, as occurs frequently in machine-type communication.

A small but efficient degree of coordination is introduced by slotted ALOHA, in which the MAC frame is divided into slots and all users transmit packets of the same length and aligned to the slots. In such a system, packets from different users may collide within a slot, although packets do not fall between two adjacent slots. Methods leveraging on the power unbalance among the colliding signals [1] or the transmission of multiple replicas of the same packet [2] have been proposed to mitigate the severe loss in throughput caused by collisions. Recently, several approaches based on multiple packet transmission and iterative interference cancellation [3]-[5] have been proposed that yield dramatic performance improvements with respect to previous solutions. The method proposed in [4] applies concepts from graph codes such as belief propagation on a packet level in order to efficiently resolve collisions. For an overview of these developments and a novel scheme for coded slotted ALOHA, we refer to [4], [6].

Another method that has been proposed recently to extract information from colliding packets, is physical layer network coding [7], [8]. In [9]-[11] the denoise-and-forward approach with channel decoding [8] has been applied in the context of random multiple access systems. An information theoretical analysis of the performance of physical layer network coding has been presented in [12]. In [13] the application and practical implementation of PNC and MUD in the multiple access channel of a WLAN for the case of two colliding signals has been presented.

In this paper, we consider a coded slotted ALOHA system and examine several options for decoding more than one packet per slot in case of a collision. In addition to the wellknown successive interference cancellation (SIC), we consider a novel scheme that comprises an additional decoding step based on network coding and compare the two with a recent scheme which employs a joint decoder [14] for all collided packets [15]. The advantage of the newly proposed scheme with respect to the one in [15] is its lower complexity, although this is achieved with a certain loss in terms of decoding probability.

In contrast to [4], we focus on the decoding within a single slot based on the received signal, i.e. on the physicallayer aspects of coded slotted ALOHA. Our approach is complementary to those that apply graph-based methods on a packet level.

\section{System Model}

We assume that a MAC frame is divided into slots of equal length and packets of $K$ users arrive synchronously. The channel is modeled as block fading, i.e. the fading coefficients $h_{k}$ are constant during one slot, but independent for each user and each slot. In addition, each user applies the same channel code of length $N$ and rate $R$. This property will turn out to be useful for the network-decoding step and for joint decoding. We denote a codeword of user $k \in\{1,2, \ldots, K\}$ by $\mathbf{c}_{k} \in \mathbb{F}_{2}^{1 \times N}$, where $\mathbb{F}_{2}$ denotes the binary Galois field. The message of user $k$ is denoted accordingly by $\mathbf{u}_{k} \in \mathbb{F}_{2}^{1 \times R N}$ and we can write for the encoding function $\mathbf{c}_{k}=\mathbf{u}_{k} \mathbf{G}$ with a common generator 
matrix $\mathbf{G} \in \mathbb{F}_{2}^{R N \times N}$. The codeword symbols $c_{k, n} \in \mathbb{F}_{2}$ are mapped to BPSK symbols $x_{k, n}=\mu\left(c_{k, n}\right) \in\{-1,1\}$, and the mapping function $\mu$ for bit vectors is defined element-wise. We chose this modulation for ease of exposition, noting that the extension to higher-order QAM is relatively straightforward and does not change the main principles. The received signal for a collision of $K$ packets can then be written as

$$
y_{n}=\sum_{k=1}^{K} h_{k} x_{k, n}+w_{n}, \quad w_{n} \sim \mathcal{N}(0,1)
$$

where the fading coefficient follows a Rayleigh distribution with $h_{k}=\left|h_{k}^{(\mathrm{c})}\right|$ for $h_{k}^{(\mathrm{c})} \sim \mathcal{C N}(0, \sqrt{\mathrm{SNR}})$. The fading coefficients are known at the receiver but not at the transmitters.

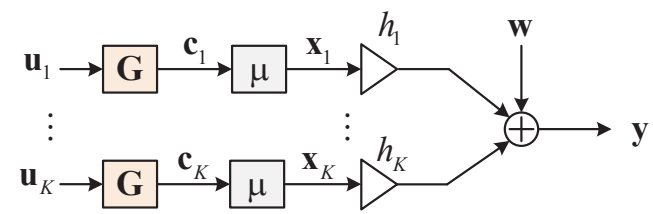

Figure 1. $K$-user multiple-access channel with block fading. All users apply the same channel code.

\section{Decoding Methods}

The receiver tries to recover as many user packets as possible per slot, based on the received signal $\mathbf{y}=\left[y_{1}, y_{2}, \ldots, y_{N}\right]$. In the following, we focus only on decoding per slot, noting that this does not exclude interference cancellation techniques with packets decoded in other slots but is complementary.

\section{A. Separate Decoding}

The simplest approach is to decode each packet separately, considering all other packets as interference. As for all other schemes to follow, we utilize the CSI of all other users and the known transmit alphabet, i.e. the QAM constellation ${ }^{1}$. With this, we can write for the log-likelihood value (L-value) of user $k$ and bit position $n$,

$$
L_{k, n} \triangleq \ln \frac{P\left[c_{k, n}=1 \mid y_{n}\right]}{P\left[c_{k, n}=0 \mid y_{n}\right]}=\ln \frac{P\left[x_{k, n}=1 \mid y_{n}\right]}{P\left[x_{k, n}=-1 \mid y_{n}\right]} .
$$

Since the received symbol $y_{n}$ depends on all symbols, we need to marginalize over all other users' symbols. For this, we define the sets $\mathcal{X}_{k}^{(b)} \triangleq\left\{\mathbf{x}=\mu(\mathbf{d}): \mathbf{d} \in \mathbb{F}_{2}^{K}, d_{k}=b\right\}$ for $b \in \mathbb{F}_{2}$, with cardinality $\left|\mathcal{X}_{k}^{(b)}\right|=2^{K-1}$. We can think of the variable $\mathbf{d}$ as the vector of the coded bits of all users at the same position, i.e. $\mathbf{d}_{n}=\left[c_{1, n}, c_{2, n}, \ldots, c_{K, n}\right]^{\mathrm{T}}$. We obtain for the L-values

\footnotetext{
${ }^{1} \mathrm{~A}$ further simplification would be to consider the interference as Gaussian noise, which would result in reduced performance and is therefore no considered here.
}

$$
\begin{aligned}
L_{k, n}= & \ln \frac{\sum_{\mathbf{x} \in \mathcal{X}_{k}^{(1)}} P\left[\mathbf{x} \mid y_{n}\right]}{\sum_{\mathbf{x} \in \mathcal{X}_{k}^{(0)}} P\left[\mathbf{x} \mid y_{n}\right]}=\ln \frac{\sum_{\mathbf{x} \in \mathcal{X}_{k}^{(1)}} p\left(y_{n} \mid \mathbf{x}\right)}{\sum_{\mathbf{x} \in \mathcal{X}_{k}^{(0)}} p\left(y_{n} \mid \mathbf{x}\right)} \\
= & \ln \frac{\sum_{\mathbf{x} \in \mathcal{X}_{k}^{(1)}} \exp \left(-\left(y_{n}-\mathbf{h}^{\mathrm{T}} \mathbf{x}\right)^{2}\right)}{\sum_{\mathbf{x} \in \mathcal{X}_{k}^{(0)}} \exp \left(-\left(y_{n}-\mathbf{h}^{\mathrm{T}} \mathbf{x}\right)^{2}\right)} \\
= & \underset{\mathbf{x} \in \mathcal{X}_{k}^{(1)}}{\operatorname{jacln}}\left\{-\left(y_{n}-\mathbf{h}^{\mathrm{T}} \mathbf{x}\right)^{2}\right\} \\
& -\underset{\mathbf{j} \in \mathcal{X}_{k}^{(0)}}{\operatorname{jacln}}\left\{-\left(y_{n}-\mathbf{h}^{\mathrm{T}} \mathbf{x}\right)^{2}\right\}
\end{aligned}
$$

where $\operatorname{jacln}\left\{x_{1}, \ldots, x_{n}\right\} \triangleq \ln \sum_{i=1}^{n} \exp \left(x_{i}\right)$ denotes the Jacobian logarithm, which can be computed recursively and for which exist computationally efficient approximations [16]. These L-values are input to a soft-input decoder, which typically is a Viterbi, a turbo or an LDPC decoder.

\section{B. Successive Interference Cancellation (SIC)}

A straightforward and well-known extension of basic singleuser decoding is SIC: if a packet $\mathbf{u}_{k^{*}}$ is successfully decoded, its corresponding codeword $\mathbf{c}_{k^{*}}$ and symbol sequence $\mathbf{x}_{k^{*}}$ are known and can be subtracted from the received signal $\mathbf{y}_{n}$, creating a multiple-access channel with $K-1$ users. This process can be repeated until decoding of all remaining packets fails. To avoid unneccessary computations, we can exploit the knowledge of the instantaneous SNRs and order the users accordingly: let $\pi$ be a permutation of $\{1,2, \ldots, K\}$ such that

$$
h_{\pi(1)} \geq h_{\pi(2)} \geq \cdots \geq h_{\pi(K)}
$$

then decoding starts with user $\pi(1)$. Apart from reducing computational complexity, this ordering is also useful to reduce the probability of undetected errors. To detect correct decoding of a packet, in general an additional error detection code, e.g. a $\mathrm{CRC}$, has to be introduced into each message $\mathbf{u}_{k}$. Since there is a non-zero probability that an erroneous decoding is not detected, the number of decoding attempts with low probability of success should be kept to a minimum.

\section{Successive Interference Cancellation with Seek \& Decode $(S I C+S \& D)$}

For a coded slotted ALOHA system, a further decoding step after SIC is possible. Assume that after the SIC procedure described above, $K-K_{1}$ packets have been correctly decoding, hence leaving $K_{1} \in\{2, \ldots, K\}$ packets for which decoding failed. In this situation, the receiver can try to decode a combined packet, which is given by the sum of two or more packets. Assume that after SIC, users $1,2, \ldots, K_{1}$ have not been decoded. Then the receiver can try to decode a subset of $\left\{1,2, \ldots, K_{1}\right\}$, e.g. given by $\mathcal{K}=\left\{k_{1}, k_{2}, \ldots, k_{\ell}\right\} \subset$ $\left\{1,2, \ldots, K_{1}\right\}$. For this subset we define the sets of constellation symbols for $\ell \geq 2$ as

$$
\mathbb{X}_{\ell}^{(b)} \triangleq\left\{\mathbf{x}=\mu(\mathbf{d}): \mathbf{d} \in \mathbb{F}_{2}^{\ell} \text { with } \sum_{i=1}^{\ell} d_{i}=b\right\}, b \in \mathbb{F}_{2}
$$


and obtain the corresponding L-values as

$$
L_{n}^{\mathcal{K}}=\ln \frac{\sum_{\mathbf{x} \in \mathbb{X}_{\ell}^{(1)}} \exp \left(-\left(y_{n}-\left[h_{k_{1}} h_{k_{2}} \cdots h_{k_{\ell}}\right] \mathbf{x}\right)^{2}\right)}{\sum_{\mathbf{x} \in \mathbb{X}_{\ell}^{(0)}} \exp \left(-\left(y_{n}-\left[h_{k_{1}} h_{k_{2}} \cdots h_{k_{\ell}}\right] \mathbf{x}\right)^{2}\right)}
$$

These L-values $L_{1}^{\mathcal{K}}, L_{2}^{\mathcal{K}}, \ldots, L_{N}^{\mathcal{K}}$ are fed to the soft-input decoder, which, if successful, finds the corresponding codeword $\sum_{k \in \mathcal{K}} \mathbf{c}_{k}$ or message $\sum_{k \in \mathcal{K}} \mathbf{u}_{k}$. Note that the sum of messages or codewords is defined in the finite field $\mathbb{F}_{2}$, which is the same as the bit-wise XOR. This concept of packet combining is closely related to inter-flow network coding and it exploits the linearity of the code, which can be seen by the relation

$$
\sum_{k \in \mathcal{K}} \mathbf{c}_{k}=\sum_{k \in \mathcal{K}} \mathbf{u}_{k} \mathbf{G}
$$

For error detection, since CRC codes are also binary linear codes, the same CRC can be used. For $K_{1}$ undecoded packets, there exist

$$
\sum_{\ell=2}^{K_{1}}\left(\begin{array}{c}
K_{1} \\
\ell
\end{array}\right)=2^{K_{1}}-K_{1}-1
$$

combinations of two and more packets, for which a decoding attempt is possible from the L-values defined by (6). With this definition, note that the subsets $\mathbb{X}_{\ell}^{(b)}$ only depend on $b$ and on the number of packets $\ell$ but not on their indices $k_{1}, \ldots, k_{\ell}$.

After successful decoding of a packet combination, a subsequent idea is to re-apply interference cancellation with the packet combination. This, however, is not directly possible since the combined codeword $\sum_{k \in \mathcal{K}} \mathbf{c}_{k}$ does not correspond to any symbol sequence $\mathbf{x}_{k}$ in (1) and the sum of codewords and symbol sequences are taken over different fields, namely $\mathbb{F}_{2}$ and $\mathbb{R}$.

However, knowledge of a combined packet $\mathbf{c}_{\mathcal{K}}$ might still be useful for another decoding attempt: the cardinality of the sets $\mathbb{X}_{\ell}^{(b)}$ can be reduced by a factor of two by introducing the additional constraint of the known combined packet. Then, the L-values can be recomputed and new decoding attempts, including $\ell=1$ for individual packets can be undertaken. This approach brings about a slight additional complexity due the constraint on the decoded combination. In this case, the sets $\mathbb{X}_{\ell}^{(b)}$ will additionally depend on $n$ and hence have to computed for each coded bit.

Example 1. For the decoding of packet combinations, we give an instructive example for the subset $\mathcal{K}=\{1,2\}$, which shows the connection to physical-layer network coding in the twoway relay channel. In Table I, the possible combinations of the coded bits $d_{1}=\hat{c}_{1, n}, d_{2}=\hat{c}_{2, n}$ are listed. From this table, we can easily find the sets

$$
\begin{aligned}
& \mathbb{X}_{2}^{(0)}=\left\{\left[\begin{array}{l}
-1 \\
-1
\end{array}\right],\left[\begin{array}{l}
1 \\
1
\end{array}\right]\right\} \\
& \mathbb{X}_{2}^{(1)}=\left\{\left[\begin{array}{c}
-1 \\
1
\end{array}\right],\left[\begin{array}{c}
1 \\
-1
\end{array}\right]\right\}
\end{aligned}
$$

Table I
TABLE FOR DEFinition OF CONSTELlation subsets $\mathbb{X}_{2}^{(0)}, \mathbb{X}_{2}^{(1)}$

\begin{tabular}{|cc|cc|c|}
\hline$d_{1}$ & $d_{2}$ & $x_{1}$ & $x_{2}$ & $d_{1}+d_{2}$ \\
\hline \hline 0 & 0 & -1 & -1 & 0 \\
\hline 0 & 1 & -1 & 1 & 1 \\
\hline 1 & 0 & 1 & -1 & 1 \\
\hline 1 & 1 & 1 & 1 & 0 \\
\hline
\end{tabular}

With (6), we obtain

$$
\begin{aligned}
L_{n}^{\mathcal{K}} & =\ln \frac{e^{-\left(y_{n}+h_{1}-h_{2}\right)^{2}}+e^{-\left(y_{n}-h_{1}+h_{2}\right)^{2}}}{e^{-\left(y_{n}+h_{1}+h_{2}\right)^{2}}+e^{-\left(y_{n}-h_{1}-h_{2}\right)^{2}}} \\
& =4 h_{1} h_{2}+\ln \frac{\cosh \left(2\left(h_{1}-h_{2}\right) y_{n}\right)}{\cosh \left(2\left(h_{1}+h_{2}\right) y_{n}\right)}
\end{aligned}
$$

which corresponds to (7) in [17].

In order to assess the performance of this scheme, we count the number of innovative packets per slot. Innovative packets are correctly decoded packets or combinations of packets which cannot be obtained by combining other correctly decoded packets. The number of innovative packets is the same as the number of linearly independent packet combinations. After successful decoding of individual packets or combinations, we build a binary matrix $\mathbf{A}$ whose rows $\mathbf{a}=\left[a_{1}, a_{2}, \ldots, a_{K}\right]$ indicate the user indices which are contained in successfully decoded combinations. For instance, if the combined packet $\mathbf{c}_{1}+\mathbf{c}_{3}+\mathbf{c}_{4}$ is correctly decoded, the corresponding row is $\mathbf{a}=[1,0,1,1,0,0]$ for $K=6$. The number of innovative packets can then be calculated as the rank of $\mathbf{A}$ in $\mathbb{F}_{2}$ arithmetic.

\section{Joint Decoding and Seek \& Decode $(J D+S \& D)$}

From (1) we can observe that the received samples $y_{n}$ depend on all coded bits $c_{k, n}$ at the same bit position but are independent of bits at other positions. The optimum decoding approach is therefore to consider the vectorial symbols $\mathbf{d}_{n} \triangleq\left[c_{1, n}, c_{2, n}, \ldots, c_{K, n}\right]^{\mathrm{T}}$ jointly. This can be done with a joint decoder which operates on the vectors $\mathbf{d}_{n}$ or on an equivalent integer representation $\bar{d}_{n}$ such that $\mathbf{d}_{n}=\operatorname{bin}\left(\bar{d}_{n}\right)$. The notation $\operatorname{bin}(b)$ denotes the binary representation of the non-negative integer $b$. For LDPC and for convolutional codes, such joint decoders are described in [14], [17]. The decoder input is given by the probability vector

$$
\mathbf{p}_{n} \triangleq\left[\begin{array}{c}
p_{n}(0) \\
p_{n}(1) \\
\vdots \\
p_{n}\left(2^{K}-1\right)
\end{array}\right] \in \mathbb{R}^{2^{K}}
$$

where

$$
p_{n}(b) \triangleq P\left[\mathbf{d}=\operatorname{bin}(b) \mid y_{n}\right] \propto p\left(y_{n} \mid \mathbf{x}=\mu(\operatorname{bin}(b))\right)
$$


for $b=0,1, \ldots, 2^{K}-1$. Let $\overline{\mathbf{x}}_{b}=\mu(\operatorname{bin}(b))$, then

$$
\mathbf{p}_{n}=\alpha\left[\begin{array}{c}
\exp \left(-\left(y_{n}-\mathbf{h}^{\mathrm{T}} \overline{\mathbf{x}}_{0}\right)^{2}\right) \\
\exp \left(-\left(y_{n}-\mathbf{h}^{\mathrm{T}} \overline{\mathbf{x}}_{1}\right)^{2}\right) \\
\vdots \\
\exp \left(-\left(y_{n}-\mathbf{h}^{\mathrm{T}} \overline{\mathbf{x}}_{2^{K}-1}\right)^{2}\right)
\end{array}\right]
$$

where $\alpha$ is a scaling factor which is irrelevant for the decoding algorithm.

The decoder output is an estimate of all messages (or equivalenty of all codewords),

$$
\hat{\mathbf{U}}=\left[\begin{array}{c}
\hat{\mathbf{u}}_{1} \\
\hat{\mathbf{u}}_{2} \\
\vdots \\
\hat{\mathbf{u}}_{K}
\end{array}\right]
$$

Making use of an error detecting code, the receiver checks all possible packet combinations, i.e. all $2^{K}-1$ non-empty subsets of $\{1,2, \ldots, K\}$ and builds the binary $\mathbf{A} \in \mathbb{F}_{2}^{\left(2^{K}-1\right) \times K}$ which indicates the correct packet combinations in each row. From this matrix, the number of innovative packets is calculated as its rank

This joint decoding approach reverses the order of the SIC+S\&D method: while in SIC+S\&D the packet combination is determined first and then a decoding attempt is carried out, joint decoding first tries to decode all packets jointly and then the receiver checks which combinations are correct.

\section{E. Complexity Considerations and Possible Combined Ap- proaches}

While in the following, we focus on the achievable performance for each of the described methods, it is worth pointing out the possible combinations and performance-complexity trade-offs. For the basic separate decoding scheme, complexity could be reduced by ordering users according to their instantaneous SNR and stop decoding after the decoding of one user has failed. This will obviously cause a slight performance loss which depends mainly on the SNR differences and on the applied coding scheme, basically on the packet length. The same idea can be applied to both SIC techniques, while for SIC+S\&D, a packet combination can be checked for linear independency before the decoding attempt. The complexity of $\mathrm{SIC}+\mathrm{S} \& \mathrm{D}$ in the worst case is proportional to $2^{K}-1$ decoding attempts. The complexity of joint decoding in the case of LDPC codes is proportional to $K \cdot 2^{K}$ for belief propagation with transform-based check-node processing [18], [19]. This complexity can be reduced by applying joint decoding after SIC and on the other hand by applying reduced-complexity decoding algorithms [20].

\section{Simulation Results}

\section{A. Single-User Performance}

The outage probability for the single-user block Rayleigh fading channel is given by

$$
p_{\text {out }}=1-\exp \left(-\frac{2^{2 R}-1}{\mathrm{SNR}}\right)
$$

This holds for the channel model (1) with $K=1$ [21, Section 5.4.1] (the factor 2 before the rate is due to the real-valued channel model). For all simulations, we use an LDPC code from the WiMAX standard [22] with code rate $R=1 / 2$, word length $N=576$ bits and message length $R N=288$ bits. This widely known code has been chosen to facilitate comparabiltiy and for the availability of a joint decoder for LDPC codes. Note that for joint decoding, a non-binary LDPC decoder in $\mathbb{F}_{q}$ with $q=2^{K}$ can be applied [23], provided the definition of the bit vector corresponds to the binary image of the Galois field elements [14], [23]. Fig. (2) shows the packet error rate (PER) for the chosen code over a block Rayleigh fading channel, in comparison with the outage probability (14). For the singleuser case, the number of successfully decoded packets per slot is simply given by $1-$ PER. For the simulations, we assumed perfect error detection which is slight simplification while for a more realistic implementation, an additional error detecting code, e.g. a 24-bit CRC, can be included.

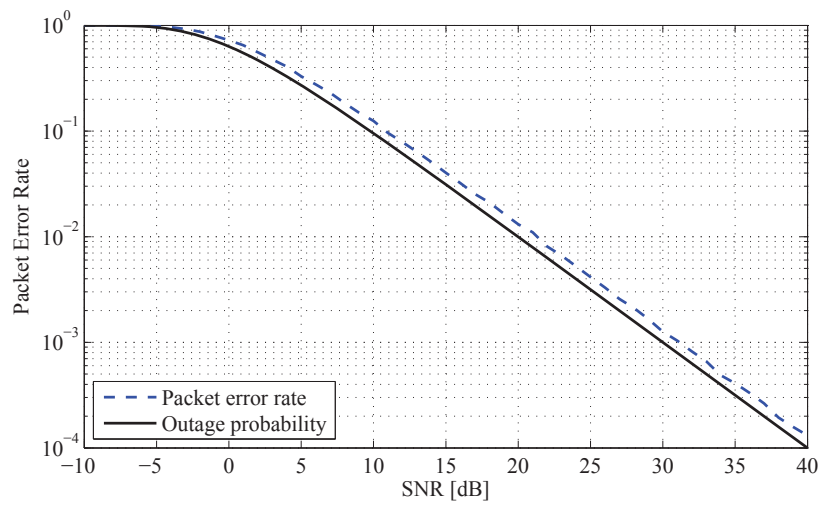

Figure 2. Outage probability and packet error rate for the single-user channel with block Rayleigh fading

\section{B. Performance for Collision Resolution}

Fig. 3 to Fig. 5 show the achieved number of innovative packets per slot with the described decoding techniques with 2, 4 and 8 users. We can see that for all cases, joint decoding performs best and its advantage increases with the number of users. For a high number of users, the advantage of joint decoding to all other techniques is dramatic. On the other hand, we point out that, unlike JD+S\&D, the SIC+S\&D scheme has the advantage that is does not require any modification at the decoder, since only the LLR calculation is modified with respect to a standard receiver. We further note that the advantage of SIC+S\&D over pure SIC decreases with 
the number of users. For sufficiently high SNR, all methods achieve benefits from collided packets, which can be most clearly seen in Fig. (3) for two users. At low SNR, the average number of recovered packets per slot is nearly identical to the single-user case while for medium to high SNR, in the mean more than one packet is recovered. For all considered cases, the number of innovative packets tends to $K$, i.e. for high SNR nearly all collided packets can be decoded.

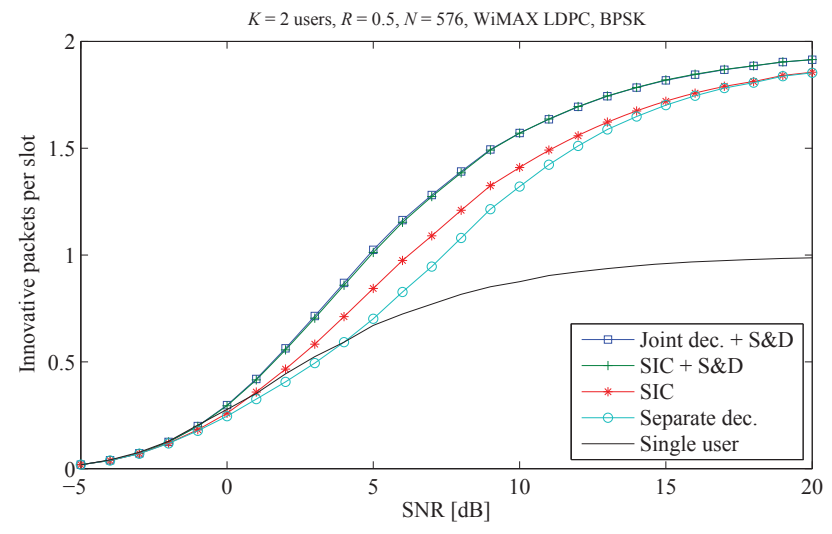

Figure 3. Two-user collision channel

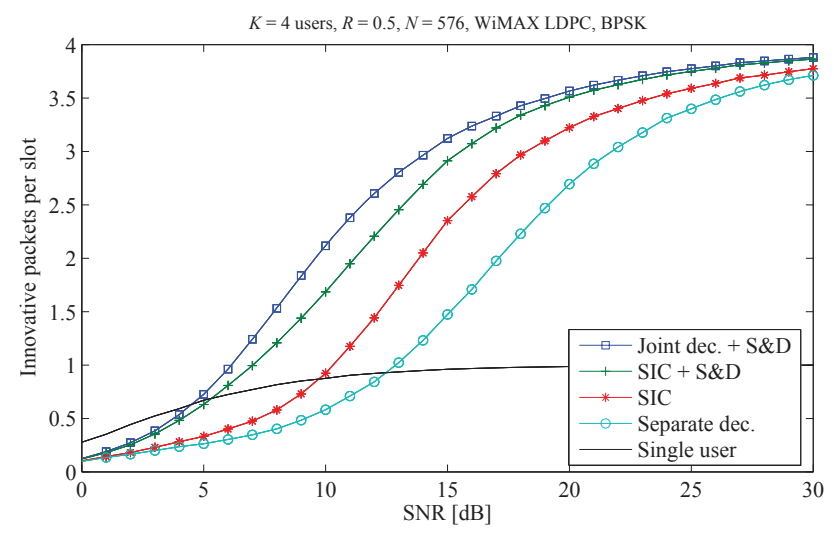

Figure 4. $K=4$

In Fig. 6, the maximum throughput for $K \in\{2,3, \ldots, 8\}$ users for each scheme is depicted. The curves are obtained by selecting for each SNR value the number of users, ranging from 2 to 8 , with the highest average number of innovative packets. We can observe that at low SNR there is little difference between the four schemes while at medium SNR, joint decoding has a significant advantage. For (very) high SNR, all schemes are limited by the maximum number of users.

\section{CONCLUSIONS}

We proposed a new scheme based on PNC and MUD that aims at retrieving innovative packets from collisions in slotted ALOHA systems. Starting from two well-known techniques,

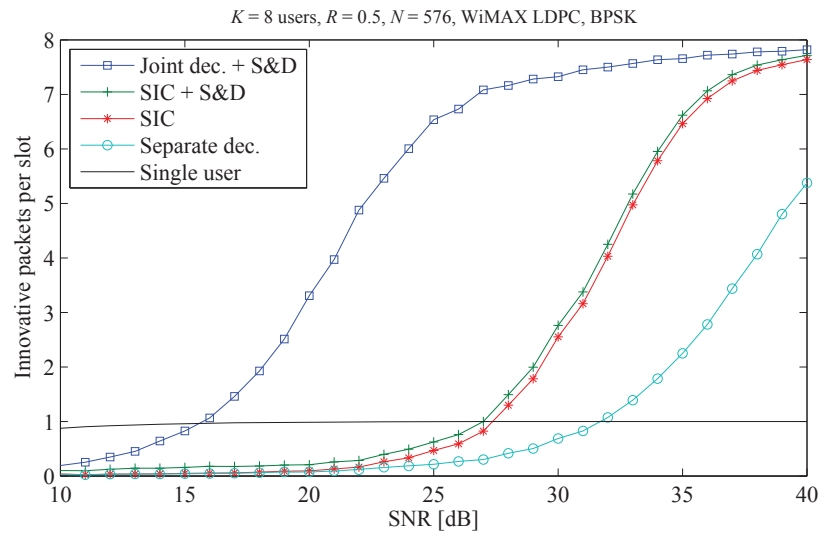

Figure 5. $K=8$

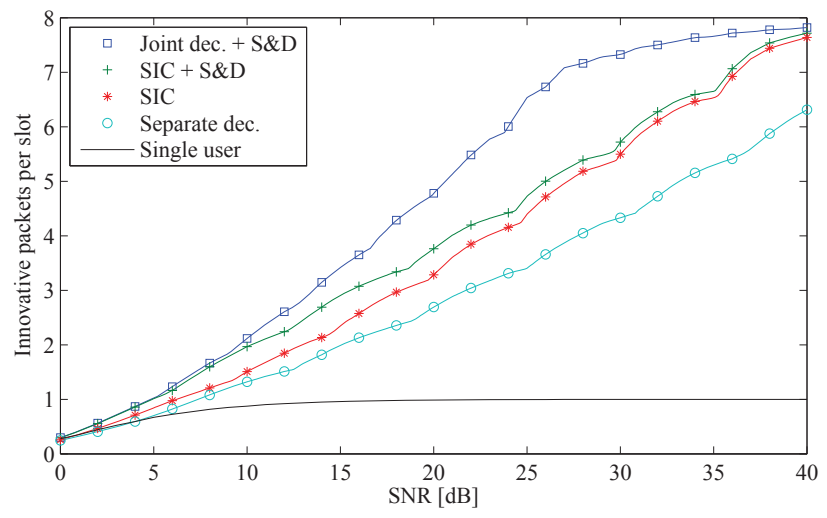

Figure 6. Maximum throughput for $K \in\{1,2, \ldots, 8\}$ for all four decoding options

namely separate decoding and successive interference cancellation (SIC), we have presented an additional decoding scheme which tries to decode the largest number of innovative packet combinations by simply modifying the detector at the receiver side, without making any modification to the channel decoder. We also evaluated the joint decoding of all collided packets and the subsequent detection of packet combinations, which constitutes the optimum approach, but is also charachteriyed by a high complexity and requires modifications at the decoder. Simulation results show that the new scheme achieves substantial gains compared to separate decoding and to SIC.

\section{ACKNOWLEDGMENT}

This work was supported by the European Commission in the framework of the FP7 Network of Excellence in Wireless COMmunications NEWCOM\# (Grant agreement no. 318306) and by the Catalan and Spanish Governments under SGR (2009SGR1046) and CICYT (TEC2011-29006-C03-01), respectively.

\section{REFERENCES}

[1] L. G. Roberts, "ALOHA packet system with and without slots and capture," SIGCOMM Computer Comm. Review, vol. 5, no. 2, pp. 28-42, April 1975. [Online]. Available: http://doi.acm.org/10.1145/1024916.1024920 
[2] G. L. Choudhury and S. S. Rappaport, "Diversity ALOHA - A random access scheme for satellite communications," IEEE Trans. Commun., vol. 31, no. 3, pp. 450-457, March 1983.

[3] E. Casini, R. D. Gaudenzi, and O. del Rio Herrero, "Contention resolution diversity slotted ALOHA (CRDSA): An enhanced random access scheme for satellite access packet networks," IEEE Trans. Wireless Commun., vol. 6, no. 4, pp. 1408-1419, April 2007.

[4] G. Liva, "Graph-based analysis and optimization of contention resolution diversity slotted ALOHA," IEEE Trans. Commun., vol. 59, no. 2, pp. 477-487, Feb. 2011.

[5] H.-C. Bui, J. Lacan, and M.-L. Boucheret, "An enhanced multiple random access scheme for satellite communications," in IEEE Wireless Telecomm. Symp. (WTS), London, UK, April 2012.

[6] E. Paolini, G. Liva, and M. Chiani, "Coded slotted ALOHA: A graphbased method for uncoordinated multiple access," IEEE Trans. Inform. Theory, Jan. 2014. [Online]. Available: http://arxiv.org/abs/1401.1626

[7] S. Zhang, S. C. Liew, and P. P. Lam, "Hot topic: Physical-layer network coding," in ACM MobiCom, Los Angeles, California, USA, Sept. 2006.

[8] P. Popovski and H. Yomo, "The anti-packets can increase the achievable throughput of a wirleless multi-hop network," in IEEE International Conference on Communication (ICC), Istanbul, Turkey, June 2006

[9] G. Cocco, C. Ibars, D. Gündüz, and O. del Rio Herrero, "Collision resolution in slotted ALOHA with multi-user physical-layer network coding," in IEEE VTC, Budapest, May 2011.

[10] G. Cocco, C. Ibars, and O. d. R. H. Deniz Gündüz, "Collision resolution in multiple access networks with physical-layer network coding and distributed fountain coding," in IEEE International Conference on Acoustics, Speech, and Signal Processing (ICASSP), Prague, May 2011.

[11] G. Cocco, N. Alagha, C. Ibars, and S. Cioni, "Network-coded diversity protocol for collision recovery in slotted ALOHA networks," Int. J. Satell. Commun. Network., Nov. 2013.

[12] J. Goseling, M. Gastpar, and J. H. Weber, "Physical-layer network coding on the random-access channel," in IEEE International Symposium on Information Theory (ISIT), July 2013, pp. 2339-2343.

[13] L. Lu, L. You, and S. C. Liew, "Network-coded multiple access," http://arxiv.org/pdf/1307.1514v1.pdf, July 2013.

[14] S. Pfletschinger, "Joint decoding of multiple non-binary LDPC codewords," in IEEE ICC Workshop on Massive Uncoordinated Access Protocols (MASSAP), Sydney, Australia, June 2014.

[15] G. Cocco and S. Pfletschinger, "Seek and decode: Random multiple access with multiuser detection and physical-layer network coding," in IEEE ICC Workshop on Massive Uncoordinated Access Protocols (MASSAP), Sydney, Australia, June 2014.

[16] P. Robertson, E. Villebrun, and P. Höher, "A comparison of optimal and sub-optimal MAP decoding algorithms operating in the log domain," in IEEE International Conference on Communication (ICC), Seattle (USA), June 1995.

[17] C. Vitiello, S. Pfletschinger, and M. Luise, "Decoding options for trellis codes in the two-way relay channel," in IEEE Workshop on Signal Processing Advances in Wireless Communications (SPAWC), Darmstadt, Germany, June 2013

[18] L. Barnault and D. Declercq, "Fast decoding algorithm for LDPC over $\mathrm{GF}\left(2^{q}\right)$," in IEEE Information Theory Workshop (ITW), Paris, France, March 2003

[19] D. Declercq and M. Fossorier, "Decoding algorithms for nonbinary LDPC codes over GF( $q$ )," IEEE Trans. Commun., vol. 55, no. 4, pp. 633-643, April 2007.

[20] A. Voicila, D. Declercq, F. Verdier, M. Fossorier, and P. Urard, "Lowcomplexity decoding for non-binary LDPC codes in high order fields,' IEEE Trans. Commun., vol. 58, no. 5, pp. 1365-1375, May 2010.

[21] D. Tse and P. Viswanath, Fundamentals of Wireless Communication. Cambridge University Press, 2005.

[22] IEEE Standard for Local and metropolitan area networks. Part 16: Air Interface for Broadband Wireless Access Systems, IEEE Standard for Local and metropolitan area networks Std., March 2005

[23] A. Goupil, M. Colas, G. Gelle, and D. Declercq, "FFT-based BP decoding of general LDPC codes over Abelian groups," IEEE Trans. Commun., vol. 55, no. 4, pp. 644-649, April 2007. 